\title{
EVITAR LA VIOLENCIA OBSTÉTRICA: MOTIVO PARA DECIDIR EL PARTO EN CASA $^{1}$
}

\author{
Dra. Teresa Ma Martínez-Mollá ${ }^{1}$, Dr. José Siles ${ }^{2}$, Dra. Ma del Carmen Solano ${ }^{3}$ \\ Autora correspondencia: Teresa $\mathrm{M}^{\mathrm{a}}$ Martínez-Mollá \\ Correo electrónico: teremarti2@hotmail.com.
}

1. Doctora. CS Santa Faz- Ayuntamiento de Alicante. Matrona.

2. Doctor. Facultad de Ciencias de la Salud Universidad de Alicante. PDI.

3. Doctora. Facultad de Ciencias de la Salud Universidad de Alicante. PDI.

Recibido: 29-11-2018 Aceptado: 10-05-2019

${ }^{1}$ Este trabajo recibió en el año 2013 una Ayuda del Centro de Estudios sobre la Mujer (CEM) de la Universidad de Alicante para la realización de tesis doctorales que contemplen la perspectiva de género. Los resultados preliminares fueron presentados como comunicación oral en el III Congreso internacional «Género, Ética y Cuidado. Visibilizando la violencia hacia las mujeres en el embarazo», realizado en Barcelona en mayo de 2018. 


\section{RESUMEN}

\section{Objetivo}

El objetivo de este estudio se centra en conocer los motivos que influyen en la decisión del parto en casa.

\section{Metodología}

Se trata de un diseño cualitativo de corte etnográfico realizado en la provincia de Alicante entre 2009 y 2014. Participaron once parejas que tuvieron al menos un parto planificado en casa. Se realizaron veintiuna entrevistas en profundidad y dos historias de vida y se obtuvieron cinco relatos escritos. Para su análisis se apoyó en la etnografía, el modelo estructural-dialéctico y el paquete informático ATLAS-ti v6.2.

\section{Resultados}

Se obtuvieron 3 categorías: valores, creencias y deseos. De los valores, cabe destacar que ellas son reivindicativas y críticas; ellos protectores, respetuosos y participativos; ellas empoderadas, confían en su intuición y capacidad para parir. Sobre el parto creen que es un proceso normal, que suele ir bien y que es un acto familiar y trascendental. Sobre el parto en casa consideran que hay menos riesgos, se respetan los derechos y el padre tiene mayor protagonismo que en el parto hospitalario. También desean seguir con la tradición y evitar la violencia obstétrica.

\section{Conclusiones}

Con su decisión pusieron de manifiesto su discrepancia con muchas creencias sobre el parto arraigadas en la sociedad y su rechazo al parto hospitalario. Cuando compararon la atención que se recibía en casa y en el hospital optaron por el parto en casa porque evitaban la violencia obstétrica y se respetaban sus creencias y valores.

Palabras clave: valores; creencias; deseos; derechos; etnografía; modelo estructural-dialéctico; parto en casa; violencia obstétrica. 


\section{ABSTRACT}

\section{Objectives}

This study aims to identify the reasons why couples prefer home over hospital birth.

\section{Methodology}

The methodology is an ethnographic qualitative research design carried out in the Alicante Province between 2009 and 2014. Eleven couples that had had at least one planned home birth took part in the study. Twenty-one in-depth interviews and two life stories were carried out and five written records were obtained. For the analysis of the results, we relied on ethnography, the structural-dialectic model, and the software package ATLAS-ti v6.2.

\section{Results}

Three categories were identified: values, beliefs, and wishes. Among the values, it was highlighted that the participants were socially committed and critical with the system. The men were protective, respectful and participative, while the women were highly empowered, confident in their intuition and their capacity to give birth. They believe that delivery is a natural process that usually proceeds normally, a family act and a meaningful moment. Concerning home birth, they consider that there is less risk, their wishes are respected, and the father has a bigger role, contrary to what happens in hospital birth. Participants wished to continue the tradition and for their rights to be respected and avoiding obstetric violence.

\section{Conclusions}

With their decision, these couples manifested their disagreement with some beliefs rooted in society and thus they refused hospital birth. When comparing the attention received both at home and at the hospital, they chose home birth to avoid obstetric violence and to have their beliefs and values respected.

Keywords: values; beliefs; wishes; rights; ethnography; structural-dialectic model; home birth; decision-making; obstetric violence. 


\section{INTRODUCCIÓN}

En la atención obstétrica, las mujeres y sus parejas tienen que tomar múltiples decisiones y, tal y como afirman Lindgren y Erlandsson (2010), una de las más importantes es elegir el lugar donde se va a producir el nacimiento. Tomar una decisión es un proceso en el que se selecciona una acción entre un conjunto de acciones posibles. Hidalgo et al. (2007) señalan que son numerosos los factores que influyen tales como el acceso a la información y distintas circunstancias personales como valores, creencias, deseos, experiencias anteriores, expectativas y temores, entre otros.

En España, cada año un pequeño número de parejas decide realizar el parto en su casa, opción sobre la que hay mucha controversia y que es rechazada por la mayor parte de la clase médica y de la sociedad. El parto planificado en el domicilio es definido por el American College of Nurses-Midwives (2010) como «la asistencia a mujeres embarazadas seleccionadas, por profesionales cualificados, dentro de un sistema que prevé la hospitalización cuando sea necesario». Y como la evidencia científica demuestra, cuando no es un primer parto, se dan mejores resultados que en el hospital (FAME, 2017).

La OMS (2014) afirma que todas las mujeres tienen derecho a una atención digna y respetuosa en el embarazo y en el parto. Según la organización Human Rights in Childbirth (2012), en esta atención se entrecruzan distintos derechos: a la dignidad, a la intimidad, a la integridad física, a la autonomía... Cuando estos derechos no se respetan, se está ejerciendo violencia obstétrica, que fue definida por primera vez en Venezuela como «la apropiación del cuerpo y procesos reproductivos de las mujeres por personal de salud, que se expresa en un trato deshumanizador, trayendo consigo una pérdida de autonomía y capacidad de decidir libremente sobre sus cuerpos y sexualidad, en un abuso de medicalización y patologización de los procesos naturales impactando negativamente en la calidad de vida de las mujeres» (Ley orgánica Derecho de las mujeres, 2007, p. 30). Según Malacalza (2017), esta violencia puede ser física o psíquica y con frecuencia es ignorada e invisibilizada (Bianco, 2015). Como afirma Araujo-Caro (2017), la violencia obstétrica reúne diversas formas de expresión del control y opresión sobre los cuerpos y las mentes de las mujeres durante su proceso obstétrico. Dado que perpetúa las relaciones de poder naturalizando el deber de obediencia y de docilidad, debe ser entendida como violencia simbólica y estructural (Al Adib et al., 2017), así como violencia institucional, 
ya que mantiene las relaciones desiguales de poder entre el personal de salud y las mujeres propia de la cultura médica hegemónica (Valdez-Santiago et al., 2013).

Debido a que en España se está produciendo un interés creciente por el parto en casa, así como un fuerte debate sobre su conveniencia, y dada la escasa producción científica española sobre esta práctica y sobre su relación con la violencia obstétrica, se plantea la necesidad de realizar esta investigación. El objetivo de este estudio es conocer los motivos que llevan a las parejas a decidir el parto en casa.

\section{MATERIAL Y MÉTODO}

La investigación es de corte cualitativo y etnográfico. El estudio se desarrolló en la provincia de Alicante, entre 2009 y 2014. Se incluyeron a mujeres que hubieran tenido al menos una vez la experiencia de tener un hijo/a en casa, independientemente del tiempo transcurrido desde que se produjera el parto. También a las parejas (marido, novio, pareja o compañero) de estas mujeres en el momento del nacimiento. Se excluyeron a las personas que no hablaran español. El muestreo fue intencional, buscando la diversidad de discursos, la heterogeneidad y la accesibilidad (Penalva y Mateo, 2006). A través de los primeros participantes, se consiguieron nuevos/as informantes con la técnica de «bola de nieve». Participaron 11 mujeres y 10 hombres, mayoritariamente con estudios universitarios y viviendo en ciudades pequeñas o pueblos en el momento en el que se produjo el parto en casa (Tabla 1).

Para obtener diferentes visiones de la misma realidad, la información se obtuvo a través de entrevistas en profundidad, historias de vida y la lectura de relatos escritos sobre su decisión del parto en el domicilio que se solicitó a los/as participantes. Se obtuvieron veintiuna entrevistas en profundidad, dos historias de vida y cinco relatos escritos, momento en el que se produjo la saturación teórica y se dejó de recolectar datos. Las entrevistas fueron llevadas a cabo en distintas localidades de la provincia de Alicante, mayoritariamente en el domicilio la persona entrevistada, entre diciembre de 2010 y marzo de 2012. Estas duraron entre 40 y 140 minutos. Para el registro de las entrevistas se utilizó la grabadora (Olimpus VN-3500PC) y posteriormente se transcribieron íntegramente y se completaron con las notas de campo. 
Tabla 1. Participantes en el estudio: características sociodemográficas.

\begin{tabular}{|c|c|c|c|c|c|c|c|}
\hline $\mathbf{N}^{\circ}$ & $\begin{array}{l}\text { NOMBRE } \\
\text { FICTICIO }\end{array}$ & SEXO & $\begin{array}{c}\text { FECHA } \\
\text { NACI- } \\
\text { MIENTO }\end{array}$ & $\begin{array}{c}\mathrm{N}^{\mathrm{a}} \mathrm{DE} \\
\text { PARTOS }\end{array}$ & $\begin{array}{c}\mathrm{N}^{\circ} \mathrm{PEC} / \\
\text { EDAD } \\
\text { 1ER PEC }\end{array}$ & PROFESIÓN/ NIVEL DE ESTUDIOS & $\begin{array}{l}\text { LUGAR DE } \\
\text { RESIDENCIA } \\
\text { CUANDO PEC }\end{array}$ \\
\hline 01 & Manoli & M & 1969 & 2 & $2 / 26$ & Fisio/Diplomatura & Ciudad \\
\hline 02 & Candela & $\mathrm{M}$ & 1958 & 2 & $1 / 26$ & Enfermera/Licenciatura & Pueblo \\
\hline 03 & Dante & $\mathrm{H}$ & 1955 & & & Enfermero/Licenciatura & Pueblo \\
\hline 04 & Álex & $\mathrm{H}$ & 1969 & & & Contable/Licenciatura & Ciudad \\
\hline 05 & Alicia & $\mathrm{M}$ & 1955 & 2 & $1 / 37$ & Administrativa/Secundaria & Pueblo \\
\hline 06 & Santi & $\mathrm{H}$ & 1960 & & & Médico/Licenciatura & Pueblo \\
\hline 07 & Amanda & $\mathrm{M}$ & 1958 & 3 & $1 / 29$ & Matrona/Diplomatura & Pueblo \\
\hline 08 & - & - & - & - & - & Declina Participar & - \\
\hline 09 & Enya & $\mathrm{M}$ & 1963 & 3 & $1 / 43$ & T. social/ Diplomatura & Pueblo \\
\hline 10 & Alberto & $\mathrm{H}$ & 1965 & & & T. social/Diplomatura & Pueblo \\
\hline 11 & Mati & M & 1960 & 3 & $1 / 29$ & Genetista/Licenciatura & Pueblo \\
\hline 12 & Mario & $\mathrm{H}$ & 1959 & & & Informático/Bachillerato & Pueblo \\
\hline 13 & Yaiza & $\mathrm{M}$ & 1973 & 2 & $1 / 36$ & Aux. Clínica/Secundaria & Pueblo \\
\hline 14 & Marvin & $\mathrm{H}$ & 1964 & & & Informático/FP2 & Pueblo \\
\hline 15 & Elvira & M & 1980 & 2 & $1 / 28$ & Antropóloga/Licenciatura & Ciudad \\
\hline 16 & Jaime & $\mathrm{H}$ & 1973 & & & Enfermero/Diplomatura & Ciudad \\
\hline 17 & Espe & M & 1972 & 2 & $1 / 39$ & Téc. Cooperación/Diplomatura & Ciudad \\
\hline 18 & Marc & $\mathrm{H}$ & 1968 & & & Prof. Universidad/Ingeniero & Ciudad \\
\hline 19 & India & $\mathrm{M}$ & 1984 & 2 & $2 / 23$ & Agricultura ecológica/FP & Pueblo \\
\hline 20 & Dacio & $\mathrm{H}$ & 1980 & & & A. ecológica/Diplomatura & Pueblo \\
\hline 21 & Ethel & M & 1979 & 3 & $1 / 34$ & Panadera/COU & Ciudad \\
\hline 22 & Pedro & $\mathrm{H}$ & 1970 & & & Prof. primaria/Diplomatura & Ciudad \\
\hline
\end{tabular}

Elaboración propia.

Para garantizar la calidad del estudio, se llevaron a cabo diversos procedimientos (Delgado et al., 2006): se utilizaron entre otras la revisión de la congruencia entre el objetivo de la investigación y la metodología propuesta, el diseño y la teoría; se usaron distintas técnicas de obtención de datos; se realizó un registro exhaustivo de la documentación y del proceso de investigación; al finalizar las entrevistas se realizó un resumen que se consensuó con las parejas entrevistadas; se remitió por correo electrónico a los/as participantes la transcripción de las entrevistas; se les presentaron los hallazgos de los análisis preliminares y de las conclusiones para oír sus opiniones; se llevó a cabo el contraste externo en el análisis por expertas en temas de género y obstetricia y se llevó a cabo la triangulación contrastando los resultados entre los distintos investigadores.

Para analizar los datos obtenidos, se realizó en primer lugar la lectura y relectura de las trascripciones textuales de las entrevistas, de los documentos escritos y de las notas de campo hasta alcanzar una estrecha familiaridad con ellas (Hammersley y Atkinson, 
1996). Posteriormente se siguieron los procedimientos marcados por la etnografía y se contó con el paquete informático ATLAS-ti v6.2, que nos permitió almacenar de forma organizada la información elaborada durante el análisis, segmentar, codificar y recuperar fragmentos significativos de nuestro material empírico y elaborar anotaciones del proceso y los resultados del análisis. Los casos de ambigüedad o desacuerdo se resolvieron mediante discusión y consenso. Igualmente se siguieron los criterios del modelo estructural-dialéctico (Siles et al., 2009), que nos permitió conocer las comparaciones que los/as participantes establecen entre la estructura hospitalaria y domiciliaria para poder tomar su decisión (Figura 1).

Figura 1. Modelo estructural-dialéctico en la decisión del parto en casa

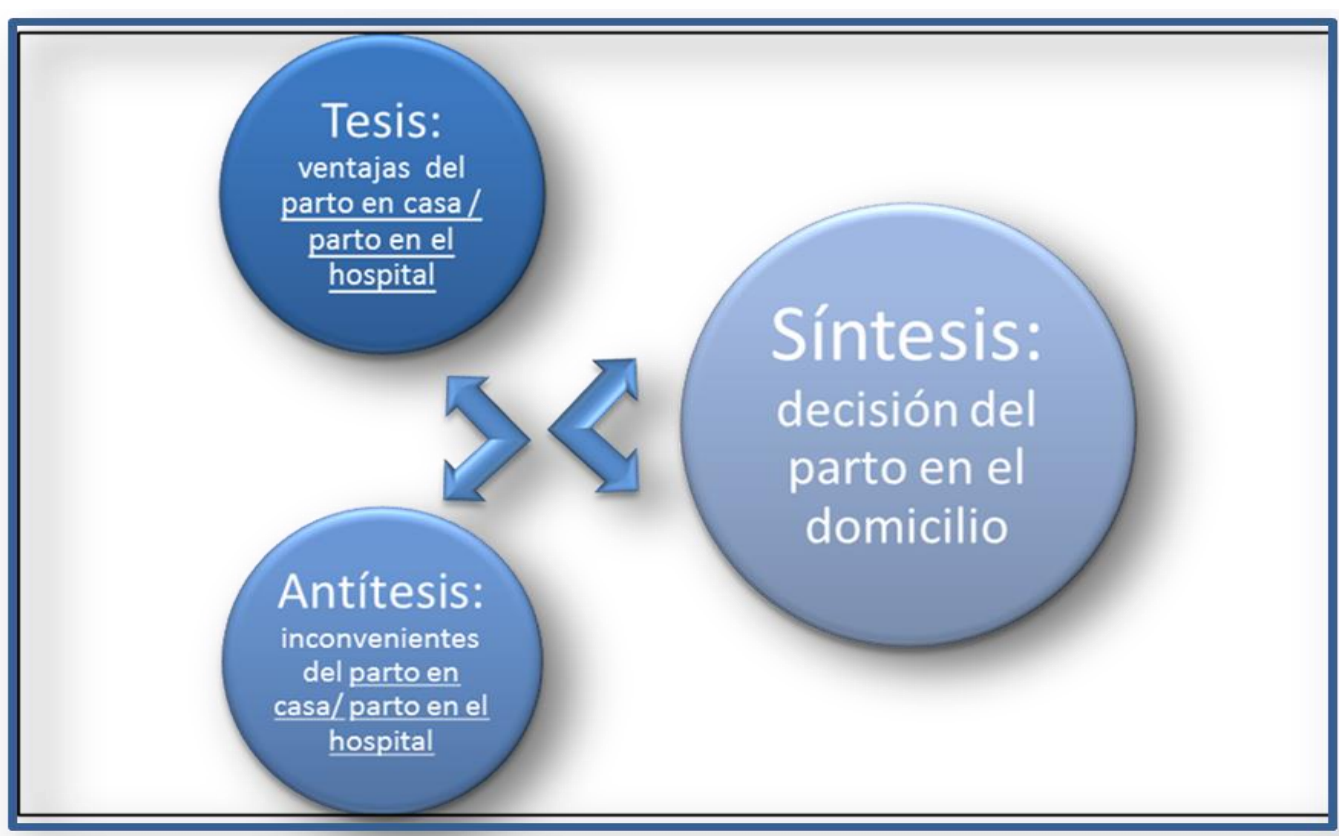

Elaboración propia.

El proyecto se presentó al comité de ética del Hospital San Juan de Alicante; se proporcionó a los/as entrevistados/as un documento de Consentimiento Informado realizado siguiendo las pautas marcadas por el RD 561/1993 (Ministerio de Sanidad y Consumo, 1993) y se tomaron las medidas necesarias para garantizar la confidencialidad y el anonimato. 


\section{RESULTADOS Y DISCUSIÓN}

\section{Análisis de los datos sociodemográficos}

Las mujeres entrevistadas, cuando tuvieron su parto en casa, eran mayoritariamente secundigestas (7 de las 11), coincidiendo con los datos de Madi y Crown (2003), al contrario que en García-Carabantes (2006) donde la mayoría eran primígestas. El nivel de estudio de las participantes es mayoritariamente de estudios universitarios, lo que coincide con los datos obtenidos por Reig-Rodríguez (2016); al contrario que Declercq y Stotland (2014) que aseguran que los niveles de estudio son comparables entre las que tienen el parto en casa o en el hospital. Resulta importante señalar que siete de los/as participantes en esta investigación tienen profesiones sanitarias, lo que supone que quizás tuvieran más facilidad para acceder a información relacionada con el parto y para contar con más elementos para ser críticos con la práctica sanitaria diaria, al igual que afirma Reig-Rodriguez (2016). De los hombres que han participado en el estudio, podemos decir que su nivel de estudios es alto, todos por encima del FPII coincidiendo con Bedwell et al. (2011). Hildingson et al. (2006) afirman que las mujeres que eligen el nacimiento en casa son mayores de 35 años, mientras que según los datos reflejados por el COIB (2010), tienen entre 25 y 35 . La edad media de nuestras participantes cuando tuvieron su primer parto en el domicilio fue de 31 años. A su vez, Anthony et al. (2005) afirman que el parto domiciliario es más frecuente en mujeres que viven en ciudades pequeñas o zonas rurales. La mayoría de las parejas entrevistadas vivían alrededor de la ciudad de Alicante, tan solo una de ellas vivió su segundo parto en una gran ciudad. Igualmente, en los países en desarrollo se realizan más partos en casa si la mujer vive en zonas rurales (Thind et al., 2008). Dado que este tipo de parto no está financiado por la sanidad pública, estas parejas cuentan con suficientes recursos económicos; al contrario de lo que sucede en los países en desarrollo, donde los nacimientos en casa son más frecuentes en situaciones de pobreza (Kruk et al., 2009).

\section{Análisis de los discursos}

Del análisis de los discursos se obtienen 3 categorías: características personales, creencias, y deseos, que a su vez se subdividen en distintas categorías que se irán desarrollando en cada uno de los apartados. 


\section{Categoría 1: características personales}

Se entiende por características personales aquellas que describen el comportamiento de un individuo tal y como lo define Amorós (2007). Como subcategorías se ha hallado que de los/as participantes se puede decir que son reivindicativos/as y críticos/as con el sistema; ellos son protectores y participativos y ellas empoderadas. Estas parejas son reivindicativas, ya que reclaman y expresan de manera vehemente qué quieren: que se respeten en todo momento sus derechos. $\mathrm{Y}$ al igual que manifiestan las mujeres entrevistadas por Boucher et al. (2009) quieren ser tratadas con dignidad, respetando su cuerpo, su tiempo, sus deseos y sus necesidades físicas, psicológicas y morales.

¡Porque también tengo derecho a tener el parto como yo quiera! (Manoli) [QU:25 32:951-1145]

Además, son críticas/os con el sistema imperante y se resisten a asumir el rol establecido para las mujeres y sus parejas en el nacimiento (Blázquez, 2009). Así, con el parto en casa muestran su rechazo a las pautas medicalizadoras del sistema médicotecnocrático y realizan un acto de resistencia y de cambio en el sistema (Cheyney, 2008).

Yo soy un poco rebelde. (Jaime) [QU:16:25 11:215-11:238]

¿Qué está sucediendo que... los partos en hospital? En nuestro entorno, será una casualidad, que mala suerte que los 15 o 20 partos que conocemos todos han tenido su historia [...]. Entonces, veíamos, claro... el excesivo intervencionismo, el querer adelantar... (Jaime) [QU:16:38 11:743-11: 1004]

Estos datos coinciden con los resultados de Hildingsson et al. (2006), donde se afirma que las mujeres suizas que tienen su parto en casa parecen tener un estilo de vida diferente al resto de la población. Estas parejas se consideran personas «alternativas» y realizan actividades que se contraponen a los modelos oficiales: medicina alternativa, antivacunas, son vegetarianas/os...

$[\ldots]$ yo era vista de una manera rara, $[\ldots]$ así, «buena chica, pero ella es así, es un poco rarita, le gustan las velas..., la homeopatía...Es así como muy natural». (Amanda) [QU:27 27:29-78] 
Las entrevistadas son mujeres empoderadas, ya que se sienten poderosas y fuertes y tienen una gran confianza en sí mismas y en su capacidad para parir, y del mismo modo opinan sus parejas:

Tenía, tal y como te decía en la entrevista, seguridad en mí misma, en mi capacidad para parir... (Amanda) [QU:22 1:504-603]

Y a la vez estábamos convencidos de que... la mujer estaba preparada fisiológicamente... para parir. (Dante) [QU:3 125 1:615-695]

Así, coincidiendo con lo observado por Lázare-Boix (2015, p. 6) son mujeres que están convencidas de que el «cuerpo femenino está preparado para asumir el parto». Cheyney (2008) relaciona este empoderamiento con el conocimiento y Lindgren (2010) añade que proviene de su propio interior. Igualmente, son mujeres que confían en su intuición más que en el asesoramiento profesional, al igual que se afirma por otros autores como Boucher et al. (2009), Cheyney (2008) y por último Declercq y Stotland (2014). Así, Amanda afirma:

Mi intuición me llevaba, me arrastraba, sin inquietud hacía allí. [QU:22 $1: 436-501]$

Los padres de este estudio asumen uno de los papeles asignados por el modelo hegemónico de masculinidad/paternidad (Figueroa y Franzoni, 2011), el de ser protectores. Seguramente, por ello, al igual que en Bedwell et al. (2011) y en MartinezMollá et al., 2015) señalan que deseaban que el parto se realizara en el lugar más seguro.

Parecía la mejor opción, y que desde luego a nivel de seguridad, no había ninguna objeción. (Jaime) [QU:15 33:588-652]

También son hombres que, de acuerdo con el modelo de nuevas masculinidades y paternidades (Figueroa y Franzoni, 2011), participan de manera más activa y buscan ser protagonistas y por lo tanto jugar un papel importante en todo el proceso reproductivo y muy especialmente en el parto:

Me sentí más útil... [...] me da la sensación de que... el... el padre ahí, es... es alguien... en el... en el parto. (Santi) [QU:6 9:1258-1414] 
Y es que, a pesar de las recomendaciones de las autoridades sanitarias, a los padres se les sigue excluyendo del parto, sobre todo en los hospitales, como se pone de manifiesto en Martínez-Mollá et al. (2013).

\section{Categoría 2: Creencias que influyen en la decisión del parto en casa}

Entendemos por creencias las ideas que se consideran verdaderas y a las que se les da crédito como ciertas (RAE, 2001). Como subcategoría encontramos creencias sobre el parto, el parto hospitalario y el parto en casa.

Así, sobre el parto creen que es normal, que suelen ir bien, que es un acto familiar y un momento trascendental. La mayoría de los/as entrevistados/as creen que el nacimiento es un proceso normal, natural o fisiológico (Boucher et al., 2009). Así, Dante afirma:

Estábamos convencidos tanto ella como yo... que el parto eh... es algo completamente natural y que no tiene por qué... ser ni manipulado ni artificial ni tal. [QU:3 8:282-352]

Pero, como señala la OMS (1996), se da la paradoja de que en el hospital todos los partos son atendidos como si fuera altamente probable la patología, aunque un 70-80 \% de los embarazos pueden ser clasificados como de bajo riesgo cuando comienza el parto.

En un hospital es... ¡ es una operación, una intervención quirúrgica! (Mati). [QU:11 13:842-933]

En esta decisión juega un papel muy importante el convencimiento que tienen estas parejas de que el parto va a ir bien. Así, el American College of Obtetricians and Ginecologist (2011) declaró que la «probabilidad real»o «riesgo absoluto» de que en el nacimiento domiciliario se produjera algún acontecimiento negativo era muy baja.

Y yo le digo: «ya, vamos a ver... si el embarazo va bien ¿por qué tiene que surgir nada?». (Alicia) [QU:5 22:516-554]

Por lo tanto, afirman que no es necesario el uso de tanta tecnología (Boucher et al., 2009; Otis y Bret, 2008) y coinciden con García-Carabantes (2006) en que un parto normal no precisa hospitalización y nos dicen:

Y ahora... todas estas moderneces de... medicalizar tanto el parto, a mí no me... no me... no me parecía. (Enya) [QU:9 1:639-746] 
Estas parejas están convencidas de que el riesgo reside sobre todo en la interferencia sobre el desarrollo natural del parto, que en numerosas ocasiones lleva a una cascada de intervenciones (Davis, 2012):

Ellos mismos con su actitud te complican el parto. (Santi) [QU:6 9:811915]

Igualmente, el parto es un asunto familiar, un momento muy íntimo que concierne a la pareja y a la familia. Así, como en Boucher et al. (2009), Candela afirma:

O lo que para nosotros era... el tema del parto, un acontecimiento muy íntimo y muy de familia... muy de pareja... [...]. ¡Solos...! ¡Solos...!

[QU:2 13:1175-1293]

A su vez, coincidiendo con Burckley (2009), dan un sentido al nacimiento, rechazando la categorización tecnocrática que lo considera un hecho exclusivamente fisiológico. Y al igual que Monoley (2009), afirman que el parto en casa proporciona a la madre y a la familia la posibilidad de vivirlo como una experiencia espiritual y trascendental que transforma a la mujer. Así lo manifiestan:

En ese momento, para nosotros jera el más importante de nuestra vida! (Candela) [QU:2 29: 1381-1481

Igualmente, son numerosas las creencias sobre el parto hospitalario y domiciliario que influyen en su decisión. Así, consideran que siempre se ha nacido en casa, aunque ahora sea infrecuente.

No me digas que es que toda la vida se ha parido en el hospital, porqué hace años la gente no paría en un hospital, mi padre nació en casa. [QU:16 58:292-439]

Sin embargo, actualmente en España y en otros países es una práctica que se sale de la norma (OMS, 2005) y, como afirma Truzzi (2017, p. 428), «queda recluida en los términos de la excepcionalidad», por lo que estas parejas, al igual que en Lindgren y Erlandsson (2011), manifiestan la percepción de sentirse diferentes: 
Y aquí éramos un poco la excepción. [...]. Lo habitual era tenerlos en

el hospital, nos salíamos un poquito de la norma. (Álex) [QU:4 6:674744]

A través del modelo estructural-dialéctico podemos analizar como estas parejas comparan las ventajas y desventajas tanto del parto hospitalario como del parto en casa y llegan a la conclusión de que el parto en casa es la mejor opción (Tabla 2).

Tabla 2. Comparación de los/as participante entre las ventajas y desventajas del parto en el hospital y el parto en casa (Modelo estructural-dialéctico).

\begin{tabular}{|c|c|c|}
\hline $\begin{array}{c}\text { DESVENTAJAS PARTO } \\
\text { HOSPITAL ( - ) }\end{array}$ & $\begin{array}{l}\text { VENTAJAS PARTO EN } \\
\text { CASA }(+)\end{array}$ & DECISIÓN \\
\hline Lo peor & Lo mejor & \multirow{16}{*}{ Parto } \\
\hline No respeto derechos & Respeto derechos & \\
\hline Violencia obstétrica & Trato digno & \\
\hline Intervencionismo & No manipulado & \\
\hline Protocolizado & Libertad & \\
\hline $\begin{array}{l}\text { Pérdida de protagonismo: } \\
\text { protagonistas los profesionales }\end{array}$ & Yo decido/protagonista & \\
\hline Ambiente hostil & $\begin{array}{l}\text { Tu ambiente / Comodidad } \\
\text { Relajado }\end{array}$ & \\
\hline Padre no & Padre protagonista & \\
\hline Profesional: el que te toca & Elijes profesional & \\
\hline Más riesgos & Seguridad & \\
\hline Miedo & Tranquilidad & \\
\hline $\begin{array}{c}\text { VENTAJAS PARTO } \\
\text { HOSPITAL }(+)\end{array}$ & $\begin{array}{c}\text { DESVENTAJAS PARTO EN } \\
\text { CASA }(-)\end{array}$ & \\
\hline En $1^{\text {er }}$ parto & Traslado al hospital & \\
\hline \multirow[t]{3}{*}{ Si hay complicaciones } & No aceptado & \\
\hline & Financiación privada & \\
\hline & Dependes de un solo profesional & \\
\hline
\end{tabular}

Elaboración propia. 
Así, están convencidas, al igual que en Boucher et al. (2009), de que en el domicilio van a poder dar al hijo/a el mejor recibimiento, mientras que en el hospital ven un gran número de inconvenientes.

Nuestra intención era mmm... darle el mayor recibimiento... (Dante) [QU:3 8:433-495]

¡Yo creo que tenía que estar penado que la gente diera a luz en los hospitales! (Candela) [QU:2 6:475-555]

Y consideran que en el domicilio se evita la violencia obstétrica, ya que se respetan sus derechos (Boucher et al. 2009; Ramírez, 2015) y afirman que se sintieron más seguras/os y más cómodos/as (Lothian, 2013).

Tomé la decisión de tener el parto en casa porque quería que se respetaran las condiciones fisiológicas del parto. [...] Con la seguridad que me da un profesional que me garantiza que el proceso va a ser respetado (Manoli). [QU:25 9:1013-1108]

Estas parejas manifiestan, al igual que en Rodríguez y Aguilera (2017), que en el hospital no siempre se ofrece un trato digno y la mujer puede llegar a sentirse abandonada y manipulada. Igualmente afirman que la atención que se recibe es impersonal (Kukulu y Öncel, 2009; Lindgren et al., 2006):

[...] en el hospital, me sentí manipulada... Yo no contaba para nada...

Todo el mundo decidía por mí. (Alicia) [QU:5 2:207-325]

Lamentan que se separe a la mujer de la pareja, de su familia y sobre todo del/a recién nacido/a, lo que se lleva a cabo sin causa médica justificada o para hacer pruebas que la evidencia demuestra que se pueden realizar posteriormente (Benítez, 2008). Así, nos dicen:

Enseguida se te llevan al niño... (Mati) [QU:11 13:966-1028]

En opinión de Dante, Candela y Amanda, en su hospital no se respetaba la intimidad, aunque ninguna de las mujeres ha manifestado, como hicieron en Kitzinger (2006), que este hecho fuera vivido como una especie de violación. 
Pues entonces nosotros no queríamos eso tan falto de (silencio), de intimidad de... ¡no sé! (Dante) [QU:3 16:962-1052]

Y coincidiendo con el informe del Observatorio de la Violencia Obstétrica (El parto es nuestro, 2016) y con lo que manifiestan las mujeres en Lessa et al. (2018), piensan que, una vez que se entra en un centro hospitalario, la atención está totalmente protocolizada y la mujer pierde su autonomía, su capacidad de decidir y su protagonismo:

Todos los partos, incluso hasta los menos complicados en un hospital tienen un protocolo. (Santi) [QU:6 5:970-1140]

Lindgren et al. (2006) señalan que en el hospital también se vulneran el principio de no maleficencia y el derecho a la integridad física por la excesiva medicalización y el alto grado de intervencionismo. Así, al igual que en Valdez-Santiago (2013), señalan que se realizan numerosas prácticas invasivas y no recomendadas como tactos repetitivos, amniorrexis, episiotomías, Kristeller, cesáreas...

Sensación de... de operación quirúrgica de alto riesgo que tienen hoy

los partos en los hospitales ¿no? (Santi) [QU:6 4:356-472]

Cabe puntualizar que todas estas prácticas por las que estas parejas sienten que sus derechos están siendo vulnerados están tipificadas como violencia obstétrica (ValdezSantiago, 2013). La institucionalización y la medicalización lleva a algunos/as de las participantes a afirmar que en el hospital hay «más riesgos» (Jackson et al., 2012) y eso puede suponer una grave amenaza para la salud (Illich, 2006). Y en contra de lo que la mayoría de la sociedad cree (Blázquez, 2009), están convencidos/as de que en los hospitales también se presentan complicaciones (Anthony et al., 2005) y de que el parto domiciliario es más seguro para la madre y el/la recién nacido/a (Boucher et al., 2009; Bedwell et al., 2011), ya que hay menos probabilidad de sufrir inducciones, episiotomías, instrumentación o cesáreas innecesarias:

¿Qué es peligroso? Pienso que hoy en día, para mí, un parto, en un hospital, creo que es más problemático. (Jaime) [QU:16 44:159-319]

Cabe señalar que estas creencias en numerosas ocasiones se apoyan en experiencias propias o de conocidas. Así, algunas de las entrevistadas manifiestan haber vivido una 
experiencia traumática en un parto hospitalario, al igual que las entrevistadas por Contreras-Tinoco (2018):

Entonces, yo lo pase ¡tan mal, tan mal!... (Mati) [QU:11 1:520-565]

Para estas parejas era fundamental poder elegir a la matrona o ginecóloga que los iba a acompañar (Boucher et al., 2009; García-Carabantes, 2006), sobre todo porque les garantizaba que se iban a respetar a lo largo de todo el proceso sus derechos, sus deseos y sus necesidades. No querían arriesgarse a depender de la suerte, de que en el hospital «te tocara» un profesional más o menos respetuoso:

Para mí era fundamental que la persona que hacía el seguimiento de mi embarazo luego tenerla en el parto, pues entonces yo también de entrada me fui a buscar a ese profesional, no me fui al sistema sanitario donde no sabía lo que me iba a encontrar. (Manoli) [QU:25 3:152-439]

De este modo, Lessa et al. (2018) señalan que se trata de mujeres que buscan una relación de igualdad con la profesional y que la consiguen en el parto en el domicilio.

Respecto al papel del padre en el parto hospitalario, coincidiendo con lo afirmado por Lindgren y Erlandsson (2011), consideran que, aunque hace años que los padres entran en el paritorio acompañando a la mujer, estos tienen poco protagonismo y en muchas ocasiones son meros espectadores y no se les permite sentirse coprotagonistas del proceso. Es por eso por lo que prefieren el parto en el domicilio, ya que pueden participar más activamente en el nacimiento (Hendrix et al., 2010).

Igualmente, reconocen que se están produciendo cambios en la atención que se da durante el parto en los hospitales, encaminados a asistir al nacimiento con un mayor respeto a los derechos y a la fisiología. Pero también creen que estos todavía son más teóricos que reales, que no hay unanimidad en la atención obstétrica y que todo depende de la voluntad del/a profesional.

Y si te tocaba alguien pues un poco enrollado pues a lo mejor te iba a tener más en consideración y si tocaba uno menos enrollado pues ¡ibas... a tragar! (Enya) [QU:9 22:899-1044]

Este hecho está corroborado en el informe La asistencia al parto de las mujeres sanas: estudio de variabilidad y revisión sistemática (Maceira et al., 2009), en el que se 
señala la existencia de una gran diferencia entre los procedimientos realizados en los distintos hospitales españoles a lo largo del año 2007.

\section{Categoría 3: Deseos que influyen en la decisión del parto en casa}

Se entiende como deseo la acción y efecto de desear. Como subcategorías se halla que estas parejas manifiestan sus deseos de seguir con la tradición y evitar la violencia obstétrica recibiendo un trato digno en el que se respetan el derecho a tomar sus propias decisiones y a la intimidad

El deseo de seguir con la tradición es el principal motivo por el que optan por el nacimiento en casa según Gebrehiwot et al. (2012). De los/as entrevistados/as, nacieron en su casa Dante, Santi, Amanda, Alberto, Mario, Yaiza, Espe, India y Dacio:

Yo había nacido en casa, ¡mi hermano había nacido en casa! Sí, bueno... de mi generación... habíamos nacido en casa, ;todos! (Dante) [QU:3 4:1220-1333]

Estas parejas desean que se respeten sus derechos a un trato digno, a tomar sus propias decisiones y a la intimidad. Y al igual que en Boucher et al. (2009), querían ser tratadas con dignidad, respetando su cuerpo, su tiempo, sus deseos y sus necesidades físicas, psicológicas y morales.

Para mí, un valor importante es la dignidad. (Alberto) [QU:28 0:08:17.17-08:24.31]

También querían ejercer su derecho de autonomía: tomar sus propias decisiones constituye uno de los principales motivos para elegir el nacimiento en casa (Lindgren et al., 2010).

Y yo decido que si tengo otro hijo no será en el hospital. (Alicia) [QU:5

2:143-204]

Para ellas, era muy importante vivir el proceso obstétrico desde su propio control y no desde el de la institución sanitaria (Montes, 2007), responsabilizarse de su proceso obstétrico (Dahlen et al., 2010) y al igual que en Boucher et al. (2009) querían ser protagonistas durante el mismo. Así, Yaiza señala:

Poder moverte por donde quieras, de... mmm, pegar un grito, llorar o patalear, o reír o hacer lo que quieras ¿no? (Yaiza) [QU:13 7:260-275] 
Además, Elvira, al igual que las mujeres en el estudio de Viisainen (2001), nos dice que en el hospital temía perder el control:

A nivel de eso, de perder tú el control, tú ya llegas y... tú ya... no..., lo que digas no... (Silencio), no cuenta ¿no?... y... no sé, era... ¡Mi mayor temor era ese!, eh, ¡fíjate! (Elvira) [QU:15 15:1015-1152]

Otro elemento al que le otorgan relevancia es que se respete su derecho a la intimidad. Afirman que deseaban vivir el parto en su hogar, donde sabían que no iban a ser observadas ni expuestas a las miradas de cualquiera (Cheyney, 2008). Así, Pieschacón (2013) señala que, en el hospital, el cuerpo de la mujer queda expuesto y se hace público lo que es íntimo. Así nos dicen:

Pero ¡yo quería estar en intimidad!, es como hacer el amor, yo el amor lo hago en intimidad, jes como hacer el amor! (Amanda) [QU:27 10:885-1003]

Para finalizar, señalamos como limitaciones de este estudio el largo tiempo que en algunas parejas ha transcurrido desde que se produjo el parto y el que no se han entrevistado a parejas que, aunque se plantearon tener el parto en casa, al final optaron por ir al hospital.

\section{CONCLUSIONES}

Los resultados de nuestro estudio avalan que los valores que estas parejas han desarrollado a lo largo de su vida junto con sus creencias sobre el proceso obstétrico y sobre la atención proporcionada en casa o en el hospital son los que los llevarán a decidir que el parto en casa es la mejor opción, en contra de la opinión mayoritaria. Consideran que esta decisión es la que más favorece sus propios deseos de que el parto sea asistido respetando en todo momento su fisiología y evitando la violencia obstétrica presente en la atención hospitalaria que se pone de manifiesto con la vulneración de los derechos a un trato digno, a la autonomía, intimidad, integridad física y no maleficencia. Igualmente, tienen la firme convicción de que el parto tiene un valor espiritual y transcendental y que el modo de nacer tiene efectos físicos y emocionales que van a tener repercusión a lo largo de toda su vida, y solo en el parto en casa se respetarán sus creencias y valores. 
Se considera que esta investigación puede ayudar a los/as profesionales a comprender los motivos que llevan a una pareja a decidir el parto en casa. Igualmente invita a reflexionar sobre la atención que se está dando en los hospitales y a modificar las prácticas medicalizadoras e intervencionistas en pro de un mayor respeto a la fisiología y a los derechos de las mujeres y sus parejas. Dado que el parto en el domicilio es un campo muy poco explorado, se podrían estudiar los factores que influyen en los/as profesionales de la obstetricia que atienden partos domiciliarios, así como la percepción que el resto de los/las ginecólogos/as y matronas tienen sobre el parto en casa.

\section{FUENTES DE FINANCIACIÓN}

Se recibió en el año 2013 una Ayuda del Centro de Estudios sobre la Mujer de la Universidad de Alicante para la realización de tesis doctorales que contemplen la perspectiva de género. 


\section{BIBLIOGRAFÍA}

Al AdIB Mendiri, Miriam, et al. La violencia obstétrica: un fenómeno vinculado a la violación de los derechos elementales de la mujer. En: Medicina legal Costa Rica. 2017, vol. 34, núm. 1, pp. 104-111. Disponible en:

$<$ www.scielo.sa.cr/scielo.php?script=sci_arttext\&pid=S1409$\underline{00152017000100104 \& \text { lang }=\mathrm{pt}>}$.

AMERICAN COLLEGE OF OBSTETRICIANS AND GYNAECOLOGISTS [Committee on Obstetric Practice]. Planned Home Birth. 2011. Disponible en:

<www.acog.org/Clinical-Guidance-and-Publications/Committee-Opinions/Committee-on-ObstetricPractice/Planned-Home-Birth?IsMobileSet=false>.

AMORós, Eduardo. Comportamiento organizacional. En busca del desarrollo de ventajas competitivas. Lima: Universidad católica Santo Toribio de Mongrovejo, 2007. Disponible en: 〈www.eumed.net/libros-gratis/2007a/231/17.htm>.

AnTHONY, S., et al. Maternal factors and the probability of a planned home birth. En: An International Journal of Obstetrics and Gynaecology. 2005, vol. 112, núm. 6, pp. 748-753.

Araujo-Cuauro, Juan Carlos. La violencia obstétrica. Una mirada desde la bioética médica a las cuestiones legales. En: Revista de Bioética Latinoamericana. 2017, vol. 20, pp. 56-76.

BeDwell, C., et al. She can choose, as long as I'm happy with it: a qualitative study of expectant fathers' views of birth place. Sexual y Reproductive Healthcare. 2011, vol. 2, núm. 2, pp. 71-75.

Benítez, G. Violencia obstétrica [Editorial]. En: Revista de la Facultad de Medicina. 2008, vol. 31, núm. 1, pp. 5-6.

BLÁZQUEZ, M $M^{\mathrm{a}}$ Isabel. Aproximación a la antropología de la reproducción. En: Revista de Antropología Iberoamericana, 2005, vol. 42, pp. 1-25. Disponible en: <www.aibr.org/antropologia/42jul/articulos/ju10506.pdf>.

BLÁZQUEZ, Ma Isabel. Ideologías y prácticas de género en la atención sanitaria del embarazo, parto y puerperio: el caso del área 12 de la Comunidad de Madrid. [Tesis doctoral]. Tarragona (España): Universidad Rovira i Virgili, 2009. Disponible en: $<$ http://tdx.cat/bitstream/handle/10803/8429/BLAZQUEZ.pdf?sequence=1 >.

BIANCO, G. La violencia obstétrica la invisibilidad de la violencia obstétrica. En: Mujer y Salud. 2015, vol. 38, pp. 36-39. 
Boucher, D., et al. Staying home to give birth: why women in the United States choose home birth. En: Journal Midwifery Women's Health. 2009, vol 54, núm 2, pp. 119126.

BURCKLEY, Sarah J. Gentle birth, gentle mothering: The Wisdom and Science of Gentle Choices in Pregnancy, Birth, and Parenting. Berkeley (California): Celestial Arts, 2009.

Cheyney, M. Homebirth as Systems-Challenging Praxis: Knowledge, Power, and Intimacy in the Birthplace. En: Qualitative Health Research. 2008, vol. 18, núm. 2, pp. 254-267.

COL·LEGI OFICIAL D'INFERMERIA DE BARCELONA [COIB]. Guía d'assistència del part a casa. Barcelona: Autor, 2010. Disponible en:

<www.coib.cat/uploadsBO/Noticia/Documents/GUIA\%20PART\%20CASA_LLAR GA.PDF>.

CONTRERAS-TinOCO, K. Violencia obstétrica en mujeres asistidas por aborto espontáneo en Guadalajara, México: expresiones de violencia institucional y autoritarismo médico. En: Musas. 2018, vol. 3, núm. 2, pp. 52-70.

Dahlen, H. G.; BARClay, L.; Homer, M. The novice birthing: theorizing first-time mothers' experiences of birth at home and in hospital in Australia. En: Midwifery. 2010, vol. 26, núm. 1, pp. 53-63.

DAVIS, E. Heart \& Hands. A midwife's guide to pregnancy and birth. New York: Ten Speed Press, 2012.

DeclercQ, E.; Stotland, N. E. Uptodate: Planned home birth. 2014. Disponible en: <www.uptodate.com/contents/planned-home-birth>.

Delgado, M. E.; VARGAS, I.; VÁZQUEZ, M. L. El rigor en la investigación cualitativa. En: Vázquez, M. L. (coord.). Introducción a las técnicas cualitativas de investigación aplicadas en salud. pp. 82-96. Barcelona: Bellaterra, 2006.

Díez, C.; Esteban, M. L. Introducción. En: VIII Congreso de Antropología. Procesos globales de cambio. Estructuras, contextos y prácticas. Simposio de Antropología del Género (Primera parte). Santiago de Compostela: Asociación Galega de Antropología, 1999, pp. 7-17.

FAME. La evidencia científica lo avala: el parto en casa es seguro [Nota de prensa]. 29 noviembre, 2017. Disponible en: 〈http://apromap.com/nota-de-prensa-fame-partoen-casa/>. 
FigueroA, J. G.; FrAnzOnI, J. Del hombre proveedor al hombre emocional: construyendo nuevos significados de la masculinidad entre varones mexicanos. En: Aguayo, Francisco; Sadler, Michelle (eds.). Masculinidades y políticas. 2011, pp. 6482. Disponible en:

<http://www.mineduc.cl/usuarios/convivencia_escolar/doc/201212041627090.2011 LibroMasculinidadesyPolIticas.pdf>.

Garcia-Carabantes, Ana Belén. Parir en casa, una realidad. Experiencia 1986-2006 En: Medicina naturista, 2006, vol. 10, pp. 592-597.

GEBREHIWOT, T., et al. Health workers' perceptions of facilitators of and barriers to institutional delivery in Tigray, Northern Ethiopia. En: BMC Pregnancy and Childbirth. 2014, vol. 14, núm. 1, pp. 137.

Hammersley, M.; AtKinson, P. Etnografía. Métodos de investigación. Barcelona: Paidós, 1994.

HENDRIX, M., et al. Differences in preferences for nulliparae between obstetric care and their partners in the Netherlands: a discrete-choice experiment. En: Journal of Psychosomatic Obstetrics and Gynecology. 2010, vol 31, núm. 4, pp. 243-251.

HidAlgO, I. M., et al. Integrando los valores y opiniones del paciente. Arboles de decisión. En: Grupo de Atención Sanitaria Basada en la Evidencia. En: Atención sanitaria basada en la evidencia. Su aplicación a la práctica clínica. Murcia: Consejería de Sanidad. 2007, pp. 449-578. Disponible en:

<www.murciasalud.es/publicaciones.php?op=mostrar_publicacionyid=103yidsec $=88$ >

HUMAN RIGHTS IN CHILDBIRTH. What are human rights in childbirth? 2012. Disponible en: 〈www.humanrightsinchildbirth.com/human-rights>.

ILLICH, I. Némesis médica. En: Borremans, V.; Sicilia, J. (eds.). Obras reunidas I. México DF: Fondo de Cultura Económica, 2006, pp. 533-763.

JACKSON, M. I.; DAHLEN, H.; SCHMIED, V. Birthing outside the system: perceptions of risk amongst Australian women who have freebirths and high-risk homebirths. En: Midwifery. 2012, vol. 28, núm. 5, pp. 561-567.

KITZINGER, Sheila. Birth as rape: There must be an end to 'just in case' obstetrics. En: British Journal of Midwifery. 2006. Vol. 14, núm. 9, pp. 544-545.

KRUK, M. E., et al. Women's preferences for place of delivery in rural Tanzania: a population-based discrete choice experiment. En: American Journal of Public Health. 2009, vol. 99, núm. 9, pp. 1666-1672. 
KUKULU, K.; Öncel, S. Factors influencing women's decision to have a home birth in rural Turkey. En: Midwifery. 2009, vol. 25, núm. 1, pp. 32-38.

LÁZARE-BoIX, S. Análisis antropológico del cuerpo en los relatos de parto normal de mujeres y profesionales de Barcelona. En: Musas. 2016 vol. 1, núm. 1, pp. 13-15.

Ley Orgánica sobre el Derecho de las Mujeres a una Vida libre de Violencia. Gaceta Oficial del 23 de abril de 2007, núm. 38.668. República Bolivariana de Venezuela. Disponible en: 〈http://venezuela.unfpa.org/doumentos/Ley_mujer.pdf>.

LESSA H. F., et al. Choosing the home planned childbirth: a natural and drug-free option. En: Rev Fun Care Online. 2018, vol. 10, núm. 4, pp. 1118-1122.

Lindgren, H.; Hildingsson, I.; Radestad, I. A. Swedish interview study: Parents' assessment of risks in home births. En: Midwifery. 2006, vol. 22, núm. 1, pp. 15-22.

LindGREN, H.; ERLANDSSON, K. Women's experiences of empowerment in a planned home birth: a Swedish population-based study. En: Birth. 2010. vol. 37, núm. 4, pp. 309-317.

LINDGREN, H. E., et al. Perceptions of risk and risk management among 735 women who opted for a home birth. En: Midwifery. 2010, vol. 26, núm. 2, pp. 163-72.

Lothian, J. A. Being safe: making the decision to have a planned home birth in the United States. En: The Journal of clinical ethics. 2013. vol. 24, núm. 3, pp. 266-275.

Maceiras, M. C.; Salgado, A.; y AtienZA, G. La asistencia al parto de las mujeres sanas: estudio de variabilidad y revisión sistemática. Madrid: Ministerio de Ciencia e Innnovación. Avalia, 2007. Disponible en:

<www.msssi.gob.es/organizacion/sns/planCalidadSNS/pdf/AsisPartoMujeresSanas. pdf>.

MADI, B. C.; CROw, R. A qualitative study of information about available options for childbirth venue and pregnant women's preference for a place of delivery. En: Midwifery. 2003, vol. 19, núm. 4, pp. 328-36.

MALACALZA, Laurana. (In)Definiciones institucionales para el abordaje de la violencia obstétrica en la provincia de Buenos Aires. En: Mora. 2017, vol. 23, núm. 1, pp. 205210.

Martinez-Molla, Teresa Ma; Solano Ruiz, Carmen; Siles Gonzalez, José. Yo acompaño: experiencia del padre en la decisión del parto en casa. En: Index de Enfermería. 2013. vol. 22, núm. 1-2, pp. 89-92. 
MARTínEZ-Mollá, Teresa, et al. Toma de decisiones del padre en la realización del parto en el domicilio. En: Investigación y Educación en Enfermería. 2015. vol. 33, núm. 3, pp. 573-583. Disponible en: 〈https://dx.doi.org/10.17533/udea.iee.v33n3a22>.

MedinA, G. Violencia Obstétrica. 2009. Disponible en: 〈www.gracielamedina.com/asset〉. Ministerio De SANIDAD y CONSUmO. Real Decreto 561/1993, de 16 de abril, por el que se establecen los requisitos para la realización de ensayos clínicos con medicamentos. Madrid: Ministerio de Sanidad y Consumo.

MolONEY, S. Female biology as sacred: Australian women's bio-spiritual experiences of menstruation and birth. [Tesis doctoral]. James Cook University, 2009. Disponible en: 〈https://researchonline.jcu.edu.au/5377/1/01Extended_abstract.pdf>.

MONTES, M. J. Las culturas del nacimiento. Representaciones y prácticas de las mujeres gestantes, comadronas y médicos. [Tesis doctoral]. Taragona: Universitat Rovira i Virgili, 2007. Disponible en: <www.tesisenxarxa.net/TDX-0607107-112247/>.

Organización Mundial de la Salud [OMS]. Cuidados en el parto normal: una guía práctica. Ginebra: OMS, 1996. Disponible en:

<http://www.holistika.net/parto_natural/oms/cuidados_en_el_parto_normal._guia_p ractica_oms.asp>.

Organización Mundial de la Salud [OMS]. Informe sobre la salud en el mundo. ;Cada niño y cada madre contarán! Ginebra: OMS, 2005. [Consulta: 20/11/2018]. Disponible en: 〈www.who.int/whr/2005/es/>.

Organización Mundial de la Salud [OMS]. Prevención y erradicación de la falta de respeto y el maltrato durante la atención del parto en centros de salud. Ginebra: OMS, 2014. Disponible en:

<www.who.int/reproductivehealth/topics/maternal_perinatal/statementchildbirth/es/>.

Organización de Naciones Unidas [ONU]. Conferencia Internacional sobre Población y Desarrollo en El Cairo. Nueva York: ONU, 1994. Disponible en: <www.un.org/spanish/conferences/accion2.htm>.

OTIS, K. E.; BRETT, J. Barreras al parto hospitalario: ¿Por qué muchas bolivianas dan a luz en casa? En: Revista Panamericana de Salud Pública. 2008, vol. 24, núm. 1, pp. 46-55.

Penalva, C.; Mateo, M. A. Tècniques cualitatives d'investigaciò. Alacant: Universitat d'Alacant. 2006. 
Pieschacón, C. F. Partería urbana en Bogotá: Construcción y reconstrucción de representaciones y prácticas durante la gestación y el parto. [Monografía de grado]. Bogotá (Colombia): Escuela de Ciencias Humanas, Universidad del Rosario, 2013. Disponible en:

<http://repository.urosario.edu.co/bitstream/handle/10336/4679/10262728372013.pdf;jsessionid=0E2ED90D8C7451757AA6178022AD8B21? sequence $=1$ > .

RAMÍREZ REYES, C. Parto en casa planificado con asistencia profesional: recuperando el protagonismo. [Tesis doctoral]. Universidad de Chile. 2015. Disponible en: < http://repositorio.uchile.cl/bitstream/handle/2250/140306/tesis\%20carla\%20ramire $\mathrm{z} \% 20 \% 281 \% 29 \% 20 \% 281 \% 29$. pdf? sequence $=1 \&$ is Allowed $=\mathrm{y}>$.

REIG RoDRíGUEZ, M. Recuperando una forma de asistencia al parto. Departamento de Enfermería Mayo, 2016. Disponible en:

<http://repositorio.uchile.cl/handle/2250/140306>.

Rodríguez Ramos, P. A.; Aguilera Ávila, L. La violencia obstétrica, otra forma de violencia contra la mujer. El caso de Tenerife. En: Musas. 2017, vol. 2, núm. 2, pp. $56-74$.

SILES, J.; GARCíA, E. Las características de los paradigmas y su adecuación a la investigación en enfermería. En: Enfermería Científica. 1995, vol. 160/161, pp. 1015.

SILES, J., et al. La tutorización de prácticas clínicas de enfermería desde la perspectiva de los profesores titulares implicados: un estudio realizado desde la teoría crítica y el modelo estructural dialéctico. En: Siles, J.; Solano, M. C. Antropología educativa de los cuidados: una etnografía del aula y las prácticas clínicas. Alicante: Marfil, 2009, pp. $163-182$

THIND, A., et al. Where to deliver? Analysis of choice of delivery location from a national survey in India. En: BMC Public Health. 2008, vol. 8, núm. 29.

TruzZI, Serena. Parir en casa. Un estudio antropológico de la atención domiciliaria al parto en el contexto andaluz. [Tesis doctoral]. Sevilla: Universidad de Sevilla, 2017. Disponible en <https://idus.us.es/xmlui/handle/11441/69709>.

VALDEZ-SANTIAGO, ROSARIO, et al. Nueva evidencia a un viejo problema: el abuso de las mujeres en las salas de parto. En: Revista CONAMED. 2013, vol. 18, núm. 1, pp. 1420. 